Reisevorbereitung für Patienten, die regelmäßig Opioide einnehmen

Zur Unterstützung von Ärzten und Apothekern bei der Reiseberatung von Patienten, die kontinuierlich Opioide einnehmen, bietet 1 A Pharma das Informationspaket "Reisen mit Opioiden" an. Darin finden sich unter anderem Hinweise zur Lagerung und zum Transport der Medikamente. Auch ein mehrsprachiger Opioidausweis sowie zwei Bescheinigungen für das Mitführen von Betäubungsmitteln im Ausland sind Teil des Pakets. Dieses enthält die Materialien jeweils in fünffacher Ausführung und ist kostenlos per Fax unter 0800/6 131111 oder unter www.1apharma.de erhältlich.

Nach Information von 1 A Pharma

\section{Servicematerial für Patienten in vier Sprachen verfügbar}

Für die Indikationen "Schmerz" sowie "Bluthochdruck" sind ab sofort Informationsmaterialien für Patienten in den Sprachen Englisch, Französisch, Türkisch und Arabisch verfügbar. Darunter sind ein Ratgeber Schmerz sowie ein Schmerztagebuch und eine Schmerzskala. Für Bluthochdruck ist neben einem Ratgeber auch ein Blutdruckpass erhältlich. Ärzte und Apotheker können die Materialien kostenlos bei $1 \mathrm{~A}$ Pharma per Fax unter 0800/6 131111 oder unter www.1apharma.de bestellen.

Nach Informationen von 1 A Pharma

\section{Morphin Aristo ${ }^{\oplus}$ akut jetzt auch als 10/20 mg Tabletten}

Seit Juli 2016 ist mit den Morphin Aristo ${ }^{\circledR}$ akut 10/20 mg Tabletten ein MorphinGenerikum in fester Form auf dem Markt. Durch die Wasserlöslichkeit der Tabletten lassen diese beispielsweise bei Schluckbeschwerden auch eine Anwendung in flüssiger Form zu. Der Wirkeintritt des ab sechs Jahren zugelassenen Medikaments beträgt bei vier- bis sechsmal täglicher Gabe 30 bis 90 Minuten. Die Wirkung hält laut Hersteller vier bis sechs Stunden an.

Nach Information von Aristo Pharma

\section{Tapentadol bei starken chronischen Rücken- schmerzen als Basistherapeutikum etabliert}

— Der chronische Rückenschmerz sei „extrem schwierig zu behandeln", da er häufig nozizeptive und neuropathische Elemente hat, betonte Professor Ralf Baron, Klinik für Neurologie, Leiter Sektion Neurologische Schmerzforschung und -therapie, Universitätsklinikum Schleswig-Holstein, Kiel. Für den Wirkstoff Tapentadol liegen viele positive Ergebnisse aus Phase-III-Studien vor, in denen Patienten mit chronischen Rückenschmerzen behandelt wurden. Welche weiteren Vorteile bietet der Einsatz in der Klinik und der Praxis?

Zugelassen wurde Tapentadol (Palexia ${ }^{\circledR}$ ) in Deutschland im Jahr 2010. In den letzten fünf Jahren hat sich das Analgetikum als ein Standardtherapeutikum in der Behandlung chronischer Schmerzen etabliert (u.a.: Baron R et al., Pain Pract 2016, 16:580-599). Die häufigsten Diagnosen für die Verschreibung sind nach Angaben von Kai Martens, Grünenthal, Stolberg, Rückenschmerzen und Osteoarthrose.

Tapentadol hat zwei synergistische Wirkmechanismen: als $\mu$-Opioid-Rezeptor-Agonist in Gehirn und Rückenmark (MOR) und als Wiederaufnahmehemmer des Noradrenalins (NRI). Laut Baron bietet der Einsatz des MOR-NRI große Flexibilität, denn für den Wirkstoff liegen positive Daten für neuropathische, nozizeptive und gemischte Schmer- zen vor. Tapentadol kennzeichne ein gutes Nebenwirkungsprofil und ein geringes Interaktionspotenzial, betonte Ulf Schutter, Schmerzspezialist am Marienhospital Marl. Der MOR-NRI sei „Goldstandard bei Spezialisten", weil

- viele Primäreinstellungen direkt nach oder bereits anstelle von t-NSAR/Coxiben

_ und/oder nichtsauren Analgetika erfolgen können,

_ die Wirtschaftlichkeit durch das Einsparen der Co-Analgetika ein Grund für die Umstellung sei,

_ die Spezialisten den Nicht-Spezialisten bei der Dosisfindung unterstützen könnten. Schutter empfahl für die individuelle Dosiseinstellung ein Titrationsschema (Abb. 1). Mittlerweile sei Tapentadol ein "wichtiger Bestandteil im Schmerz-Wirkstoff-Koffer" des Hausarztes, berichtete Jörg Werner Piroth, niedergelassener Hausarzt, Aachen. Dank der Rabattverträge sei eine wirtschaftliche Versorgung gesichert. Als Erleichterung für den Einsatz schätzt Piroth die Möglichkeit ein, den Wirkstoff bei Bedarf schnell auftitrieren zu können.

Dr. Friederike Holthausen

Geburtstags-Pressegespräch " 5 Jahre - einfach Palexia: Therapie chronischer Schmerzen in Klinik und Praxis", Düsseldorf. Veranstalter: Grünenthal $\mathrm{GmbH}$, Aachen.

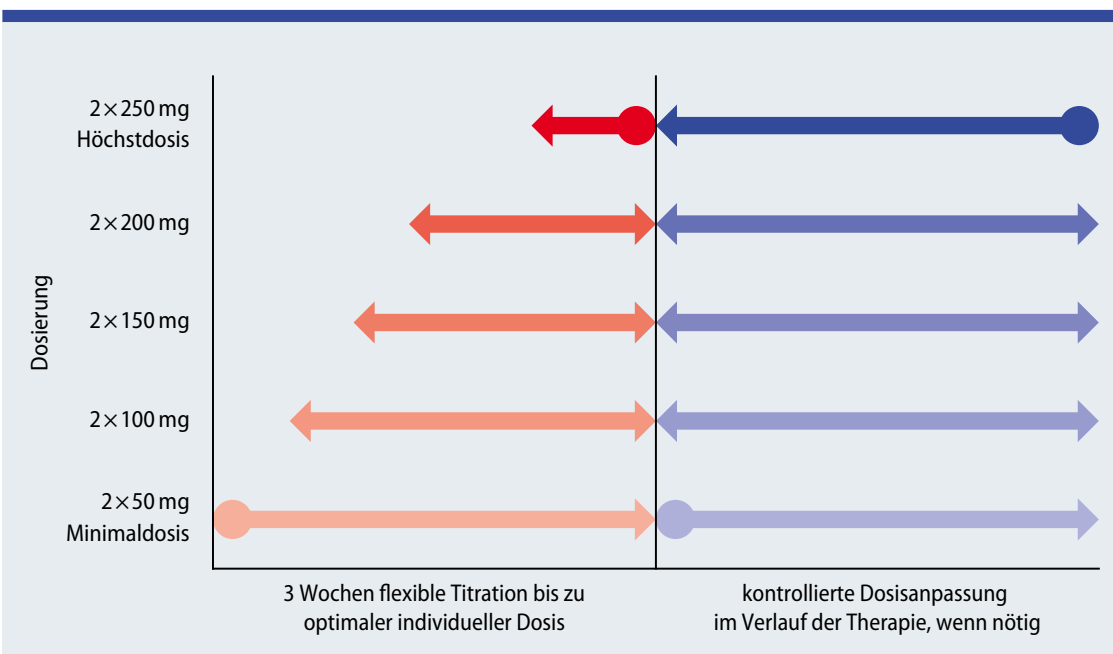

Abb. 1: Titrationsschema für Tapentadol (nach Ulf Schutter, Schmerzspezialist am Marienhospital, Marl) 